\title{
Three-Jet Production in Electron-Positron Collisions at Next-to-Next-to-Leading Order Accuracy
}

\author{
Vittorio Del Duca, ${ }^{1, *}$ Claude Duhr, ${ }^{2,3, \dagger}$ Adam Kardos, ${ }^{4}$ Gábor Somogyi, ${ }^{4}$ and Zoltán Trócsányi ${ }^{4}$ \\ ${ }^{1}$ Institute for Theoretical Physics, ETH Zürich, 8093 Zürich, Switzerland \\ ${ }^{2}$ CERN Theory Division, 1211 Geneva 23, Switzerland \\ ${ }^{3}$ Center for Cosmology, Particle Physics and Phenomenology (CP3), \\ Université Catholique de Louvain, 1348 Louvain-La-Neuve, Belgium \\ ${ }^{4}$ University of Debrecen and MTA-DE Particle Physics Research Group, H-4010 Debrecen, PO Box 105, Hungary
}

(Received 7 April 2016; published 7 October 2016)

\begin{abstract}
We introduce a completely local subtraction method for fully differential predictions at next-to-next-toleading order (NNLO) accuracy for jet cross sections and use it to compute event shapes in three-jet production in electron-positron collisions. We validate our method on two event shapes, thrust and $C$ parameter, which are already known in the literature at NNLO accuracy and compute for the first time oblateness and the energy-energy correlation at the same accuracy.
\end{abstract}

DOI: $10.1103 /$ PhysRevLett.117.152004

One of the most important fundamental parameters in the standard model is the strong coupling $\alpha_{s}$. A clean environment for determining $\alpha_{s}$ is the study of event shape distributions in $e^{+} e^{-}$collisions [1]. Indeed, while at leading order (LO) the production of two jets is a purely electroweak process, the dominant contribution to the production rate of every additional jet in the final state is directly proportional to the strong coupling. Since the initial state does not involve colored partons, nonperturbative QCD corrections are restricted to hadronization and power corrections affecting the final state configuration. These corrections can be determined either by extracting them from data by comparison to Monte Carlo predictions or by using analytic models. The precision of the theoretical predictions is thus mostly limited by the accuracy in the perturbative expansion in the strong coupling. Currently, the state of the art includes next-to-leading order (NLO) predictions for the production of up to five jets [2-4] (and up to seven jets in the leading color approximation [5]), and next-to-next-to-leading order (NNLO) predictions for the production of three jets [6,7]. Moreover, fixed-order predictions can be matched to resummation calculations (see examples in Ref. [8]), which take into account classes of logarithmically enhanced contributions to all orders in perturbation theory.

The goal of this Letter is twofold. First, we present a framework to compute fully differential predictions at NNLO accuracy for processes with a colorless initial state and involving any number of colored massless particles in the final state. Second, we apply our method to event shape

Published by the American Physical Society under the terms of the Creative Commons Attribution 3.0 License. Further distribution of this work must maintain attribution to the author(s) and the published article's title, journal citation, and DOI. observables with at least three hard final-state partons. As our framework allows for a fully differential description of the final state, it puts no restriction beyond infrared safety on the class of observables that we can consider. The cornerstone of our framework is the completely local subtractions for fully differential predictions at next-tonext-to-leading order (CoLoRFulNNLO) method to regularize infrared divergences [9]. The method is based on the universal factorization of QCD matrix elements in soft and collinear limits. It takes into account all spin and colorcorrelations among the final-state particles and as a result the subtractions are completely local.

Predictions at NNLO in perturbative QCD generically require the computation of two-loop corrections to the Born process, as well as one-loop and tree-level contributions to the processes with one or two additional partons in the final state. The two-loop matrix elements for $\gamma^{*} / Z \rightarrow q \bar{q} g$ have been computed in Ref. [10]. The one-loop corrections to four-jet production have been computed in Ref. [11], and the tree-level matrix elements for the production of five jets were obtained for first time in Ref. [12]. The sum of these contributions is finite for infrared-safe observables, but taken separately, they all exhibit explicit divergences coming from loop integrations and/or implicit divergences when one or more partons in the final state are unresolved. Thus each contribution needs to be separately rendered finite in four dimensions before any numerical computation can be performed.

The CoLoRFulNNLO method deals with this issue by using universal counterterms to redistribute divergences between different final-state multiplicities. In every singular region of phase space we subtract the corresponding infrared divergence through a suitably constructed approximate matrix element. As different singular regions overlap, a careful bookkeeping is required in order to avoid double 
counting when subtracting the divergences. Moreover, within our framework beyond NLO, we also need to consider iterated singular limits where partons become successively unresolved. Finally, spin correlations in gluon decay are retained, and the counterterms are fully local in phase space. In the CoLoRFulNNLO framework the distribution of the NNLO correction to an observable $J$ can be written as a sum of three contributions, each being separately finite in $d=4$ dimensions,

$\sigma^{\mathrm{NNLO}}[J]=\int_{m+2} d \sigma_{m+2}^{\mathrm{NNLO}}+\int_{m+1} d \sigma_{m+1}^{\mathrm{NNLO}}+\int_{m} d \sigma_{m}^{\mathrm{NNLO}}$,

where

$$
\begin{aligned}
d \sigma_{m+2}^{\mathrm{NNLO}}= & \left\{d \sigma_{m+2}^{\mathrm{RR}} J_{m+2}-d \sigma_{m+2}^{\mathrm{RR}, A_{2}} J_{m}\right. \\
& \left.-\left[d \sigma_{m+2}^{\mathrm{RR}, A_{1}} J_{m+1}-d \sigma_{m+2}^{\mathrm{RR}, A_{12}} J_{m}\right]\right\}_{d=4}, \\
d \sigma_{m+1}^{\mathrm{NNLO}}= & \left\{\left[d \sigma_{m+1}^{\mathrm{RV}}+\int_{1} d \sigma_{m+2}^{\mathrm{RR}, A_{1}}\right] J_{m+1}\right. \\
& \left.-\left[d \sigma_{m+1}^{\mathrm{RV}, A_{1}}+\left(\int_{1} d \sigma_{m+2}^{\mathrm{RR}, A_{1}}\right)^{\mathrm{A}_{1}}\right] J_{m}\right\}_{d=4}, \\
d \sigma_{m}^{\mathrm{NNLO}}= & \left\{d \sigma_{m}^{\mathrm{VV}}+\int_{2}\left[d \sigma_{m+2}^{\mathrm{RR}, A_{2}}-d \sigma_{m+2}^{\mathrm{RR}, A_{12}}\right]\right. \\
& \left.+\int_{1}\left[d \sigma_{m+1}^{\mathrm{RV}, A_{1}}+\left(\int_{1} d \sigma_{m+2}^{\mathrm{RR}, A_{1}}\right)^{\mathrm{A}_{1}}\right]\right\}_{d=4} J_{m} .
\end{aligned}
$$

$J_{n}$ denotes the value of the infrared-safe observable $J$ evaluated on a final state with $n$ resolved partons. The various subtraction terms have been defined explicitly and their integrals over the factorized phase space of the unresolved partons have been obtained in Ref. [9].

Equation (2) includes the double-real (RR) contribution that exhibits singularities whenever one or two partons become unresolved. We subtract from this an approximate cross section $d \sigma_{m+2}^{\mathrm{RR}, A_{2}}$ which has the same singularities in the doubly unresolved limits as the RR matrix element. The difference is still singular in singly unresolved regions and an additional counterterm is needed. To that effect, we subtract the quantity $d \sigma_{m+2}^{\mathrm{RR}, A_{1}}$, and compensate for the overlap between the singly and doubly unresolved regions through the term $d \sigma_{m+2}^{\mathrm{RR}, A_{12}}$.

Similarly, Eq. (3) describes the emission of one additional parton at one loop, the real-virtual (RV) contribution. In addition to explicit infrared poles coming from the loop integration, the RV contribution has kinematic singularities when this additional parton is soft or collinear to another colored particle. These are regularized by the approximate one-loop cross section $d \sigma_{m+1}^{\mathrm{RV}, A_{1}}$. The difference is now free of implicit singularities, but the explicit infrared singularities are still present. These poles are the same as the singly unresolved singularities in $d \sigma_{m+2}^{\mathrm{RR}, A_{1}}$ in integrated form, and they cancel once the corresponding term is added back. The integral of $d \sigma_{m+2}^{\mathrm{RR}, A_{1}}$, however, still has one-parton singularities, which are regularized by the last term in Eq. (3).

The last contribution to the NNLO distribution, shown in Eq. (4), includes two-loop virtual (VV) corrections to the LO process. The two-loop integrations lead to explicit infrared singularities. In Eqs. (2) and (3) we had introduced five counterterms, but so far only $d \sigma_{m+2}^{\mathrm{RR}, A_{1}}$ has been added back in integrated form. The explicit two-loop singularities cancel against the phase-space singularities of the remaining four counterterms, which are shown explicitly in integrated forms in Eq. (4). We computed all terms in these integrated forms that become singular in $d=4$ dimensions and demonstrated the cancellation of these divergences analytically. We also computed analytically the logarithmic terms in the finite part of the integrated subtractions that become singular on the edges of the phase space, while we evaluated the rest of the finite part of the integrated subtractions numerically. We add the uncertainty of these numerical evaluations to the uncertainty of the Monte Carlo integration of the $n$-parton integrations in Eq. (1) in quadrature.

CoLoRFulNNLO has already been successfully applied to compute NNLO corrections to differential distributions describing the decay of a Higgs boson into a pair of $b$ quarks [13]. Here we apply this framework for the first time to the computation of NNLO observables with more than two colored partons in the final state. In particular, we consider event-shape observables in $e^{+} e^{-} \rightarrow \gamma^{*} \rightarrow 3$ jets and we study NNLO corrections to them. If $\mathcal{O}$ denotes a generic event shape observable, we write

$\frac{1}{\sigma_{0}} \frac{d \sigma}{d \mathcal{O}}=\frac{\alpha_{s}}{2 \pi} A(\mathcal{O})+\left(\frac{\alpha_{s}}{2 \pi}\right)^{2} B(\mathcal{O})+\left(\frac{\alpha_{s}}{2 \pi}\right)^{3} C(\mathcal{O})+O\left(\alpha_{s}^{4}\right)$,

where $\sigma_{0}$ is the leading-order prediction for the process $e^{+} e^{-} \rightarrow$ hadrons in perturbation theory. In this Letter we concentrate on four event shapes. The first two, thrust $[14,15]$ and $C$ parameter [16,17], have already been studied at NNLO accuracy $[6,7]$ and serve as a validation of our method. The other two, oblateness and energy-energy correlation, have never been presented at NNLO accuracy and constitute our main phenomenological results. Thrust is defined as

$$
T=\max _{\vec{n}}\left(\frac{\sum_{i}\left|\vec{n} \cdot \vec{p}_{i}\right|}{\sum_{i}\left|\vec{p}_{i}\right|}\right),
$$

where $\vec{p}_{i}$ denote the three-momenta of the partons and $\vec{n}$ defines the direction of the thrust axis, $\vec{n}_{T}$, by maximizing 
the sum on the right-hand side over all directions of the final-state particles. In order to define oblateness, we need two variants of this definition, thrust major and thrust minor. Thrust major is given by

$$
T_{M}=\max _{\vec{n} \cdot \vec{n}_{T}=0}\left(\frac{\sum_{i}\left|\vec{n} \cdot \vec{p}_{i}\right|}{\sum_{i}\left|\vec{p}_{i}\right|}\right),
$$

where $\vec{n}$ defines the direction of the thrust-major axis, $\vec{n}_{T_{M}}$, by maximizing the sum on the right-hand side over all directions orthogonal to the thrust axis. Similarly,

$$
T_{m}=\frac{\sum_{i}\left|\vec{n}_{T_{m}} \cdot \vec{p}_{i}\right|}{\sum_{i}\left|\vec{p}_{i}\right|}, \quad \text { with } \quad \vec{n}_{T_{m}}=\vec{n}_{T} \times \vec{n}_{T_{M}},
$$

defines thrust minor, where the thrust-minor axis, $\vec{n}_{T_{m}}$, is orthogonal to both the thrust and thrust-major axes. Oblateness $O$ is then the difference of thrust major and thrust minor [18],

$$
O=T_{M}-T_{m} .
$$

The value of the $C$ parameter for massless final-state particles is

$$
C_{\mathrm{par}}=\frac{3}{2} \frac{\sum_{i, j}\left|\vec{p}_{i}\right|\left|\vec{p}_{j}\right| \sin ^{2} \theta_{i j}}{\left(\sum_{i}\left|\vec{p}_{i}\right|\right)^{2}}
$$

where $\theta_{i j}$ is the angle between $\vec{p}_{i}$ and $\vec{p}_{j}$.

Finally, energy-energy correlation [19] is the normalized energy-weighted cross section defined in terms of the angle between two particles $i$ and $j$ in an event,

$\operatorname{EEC}(\chi)=\frac{1}{\sigma_{\text {had }}} \sum_{i, j} \int \frac{E_{i} E_{j}}{Q^{2}} d \sigma_{e^{+} e^{-} \rightarrow i j+X} \delta\left(\cos \chi+\cos \theta_{i j}\right)$,

where $Q^{2}$ is the squared center-of-mass energy, $E_{i}$ and $E_{j}$ are the particle energies, $\theta_{i j}=\pi-\chi$ is the angle between the two particles, and $\sigma_{\text {had }}$ is the total hadronic cross section. Experience shows that computing radiative corrections to the distributions of $C$ parameter, oblateness, and energy-energy correlations is numerically more challenging than for other three-jet event shapes.

As a validation of our method, we show in Figs. 1 and 2 the third-order coefficient in Eq. (5) for $\mathcal{O}=\tau \equiv 1-T$ and $\mathcal{O}=C_{\text {par }}$. We observe a very good numerical convergence of our method at NNLO: the absolute uncertainties of the integrations are shown as shaded narrow bands around the solid line on the upper panels (hardly visible) and the relative ones around the lines at one on the lower panels of Figs. 1 and 2. We compare our results to the predictions of Refs. [6,7] and we find agreement over a large range of $\tau$ and $C$ parameter. As the predictions published in Ref. [7] are affected by an issue with the generation of phase space in the code used to compute those results [20], we make

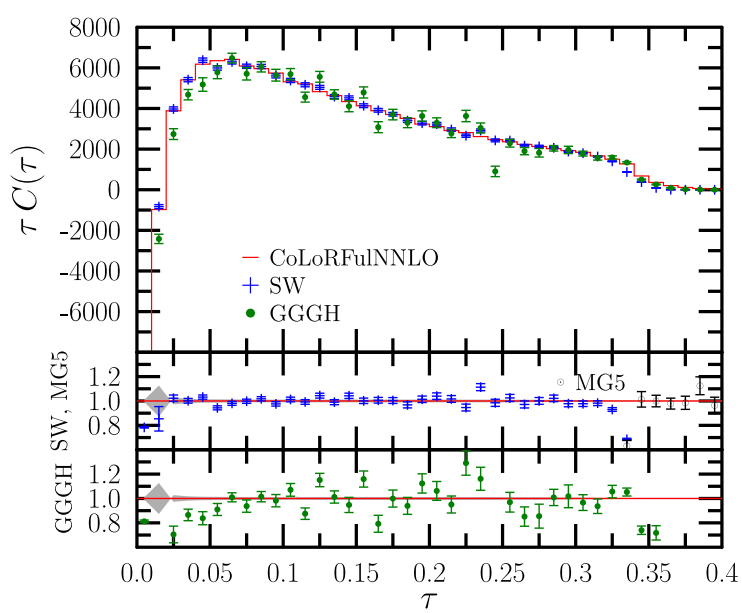

FIG. 1. The NNLO coefficient of the weighted $\tau=1-T$ distribution. The lower panels show the predictions of Ref. [7], denoted as SW, (middle panel) and those of Ref. [6], denoted as GGGH, (lower panel) normalized to ours, as well as the relative uncertainties of the numerical integrations (shaded band around the line at one). Also shown in the middle panel are the predictions of MadGraph5 aMC@NLO, denoted as MG5, for $\tau>1 / 3$.

comparisons to updated but unpublished results provided to us by $\mathrm{S}$. Weinzierl. We observe statistically significant differences beyond the kinematical limits $(\tau=1 / 3$ and $C_{\text {par }}=3 / 4$ ) where the three-particle final states vanish and the event shapes are determined by a four-jet final state. In these regions the $C(\mathcal{O})$ coefficients are determined by the NLO corrections to four-jet production, which have been known for a long time $[2,3]$ and can also be computed with modern automated tools, such as MadGraph5_aMC@NLO [21]. We have checked that our predictions are in complete agreement with those of Ref. [3] for the $C$ parameter and also with MadGraph5 aMC@NLO for both

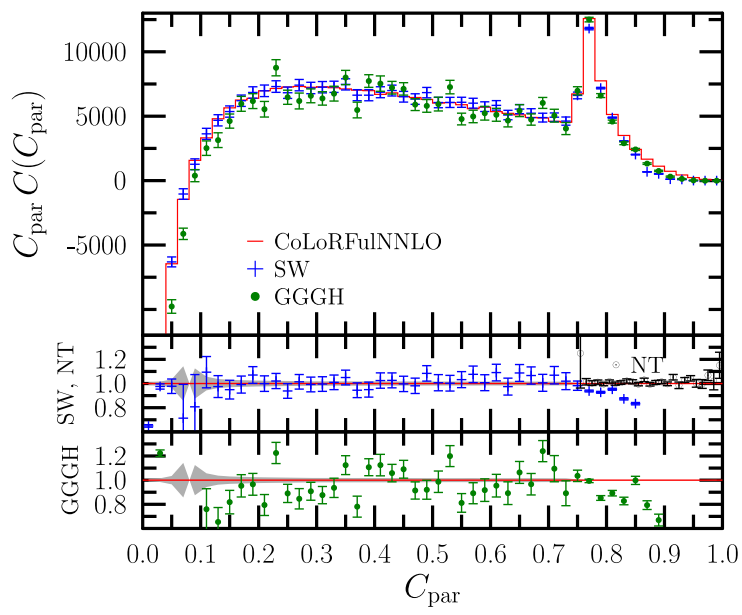

FIG. 2. The same as Fig. 1 for the $C$ parameter, with the middle panel showing also the predictions of Ref. [3], denoted as NT, for $C_{\text {par }}>3 / 4$. 


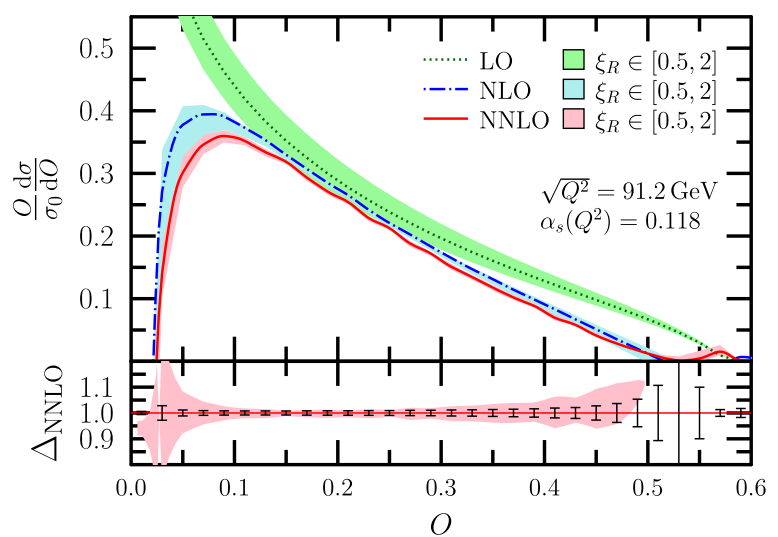

FIG. 3. Weighted distributions of oblateness $O$ at LO, NLO, and NNLO accuracy in perturbative QCD. The bands represent the dependence on the renormalization scale varied in the range $\xi_{R} \equiv \mu / \mu_{0} \in[0.5,2]$ around the default scale $\mu_{0}=\sqrt{Q^{2}}$. The lower panel shows the relative scale dependence (band) at NNLO accuracy and the relative uncertainty of the integrations (error bars).

thrust and $C$ parameter. This can be seen on the middle panels of Figs. 1 and 2 where we show the results of MadGraph 5 aMC@NLO for $\tau>1 / 3$ and those of Ref. [3] for $C_{\text {par }}>3 / \overline{4}$ normalized to ours.

We present predictions for the distributions of oblateness $O$ and energy-energy correlation EEC at NNLO accuracy in perturbative QCD for collider energy $\sqrt{Q^{2}}=91.2 \mathrm{GeV}$ in Figs. 3 and 4. The bands represent the dependence of the predictions on the renormalization scale varied in the range $[0.5,2]$ times our default scale: the total center-of-mass energy. We use $\alpha_{s}=0.118$ for the central value and the three-loop running of the strong coupling for computing the scale variations. The lower panels show the relative scale dependence of the NNLO predictions and the relative uncertainties of the integrations. Both oblateness and energy-energy correlation are known to vanish in the dijet limit. Moreover, oblateness is expected to vanish also for

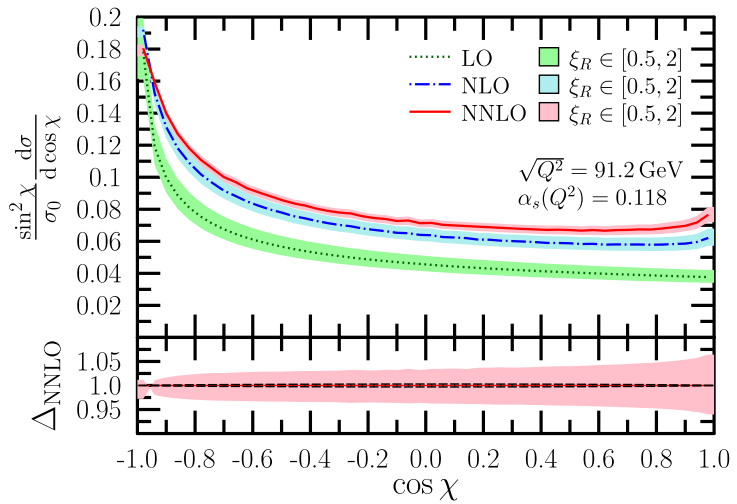

FIG. 4. Distributions of energy-energy correlation EEC at LO, NLO, and NNLO accuracy in perturbative QCD. The bands and the lower panel are like in Fig. 3.

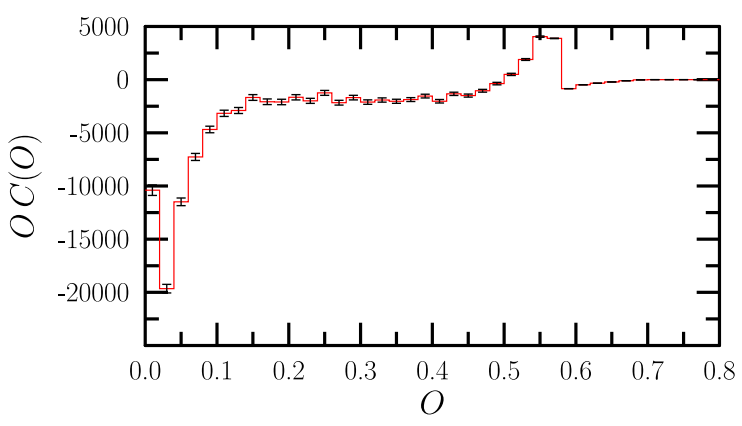

FIG. 5. Distribution of the NNLO coefficient for oblateness $O$. The error bars represent the statistical uncertainty of the Monte Carlo integrations.

cylindrically symmetric final states, while for three-parton events one has $0 \leq O \leq 1 / \sqrt{3}$. Indications of these features are visible in Figs. 3 and 4.

We observe that the NNLO corrections slightly lower, and also slightly modify the shape of the $O$ distribution compared to NLO, while the NNLO corrections enhance the EEC distribution almost uniformly. The changes in the shapes of the distributions due to the NNLO corrections can be appreciated better by looking at the distributions of the NNLO coefficients directly, as shown in Figs. 5 and 6. Also for these distributions, we observe good numerical convergence of our code.

We conclude by commenting on the behavior of the distributions corresponding to small values of the event shapes. Those regions are dominated by kinematical configurations where one of the three partons is unresolved, resulting in logarithmically enhanced contributions. In order to obtain reliable predictions the large logarithms must be resummed to all orders in perturbation theory, which is beyond the scope of the present study.

In this Letter we have introduced the CoLoRFulNNLO method to compute NNLO radiative corrections for processes with colorless initial states. We have applied it to obtain precise predictions for event shape distributions in three-jet production in $e^{+} e^{-}$collisions. We observe very good numerical convergence of our predictions over the whole range of values of the event shapes. We emphasize

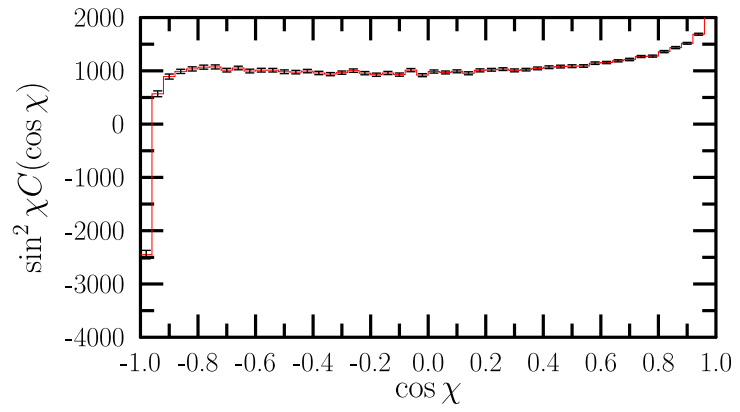

FIG. 6. Same as Fig. 5 for energy-energy correlation. 
that our framework is not restricted to three-jet production, but it can be easily extended to study differential distributions for four or more jet production once the corresponding two-loop amplitudes become available. Finally, it will be interesting to study the effects of power corrections and hadronization on our results and to compare the NNLO distributions of $O$ and EEC to data, thereby providing new observables from which the value of the strong coupling $\alpha_{s}$ can be extracted to NNLO accuracy.

This research was supported by the Hungarian Scientific Research Fund Grant No. K-101482 and by the ERC Starting Grant "MathAm." A. K. gratefully acknowledges financial support from the Post Doctoral Fellowship programme of the Hungarian Academy of Sciences and the Research Funding Program ARISTEIA, HOCTools (co-financed by the European Union (European Social Fund ESF).

* On leave from INFN, Laboratori Nazionali di Frascati, Italy. ${ }^{\dagger}$ On leave from the "Fonds National de la Recherche Scientifique" (FNRS), Belgium.

[1] Z. Kunszt, P. Nason, G. Marchesini, and B. R. Webber, in Proceedings, Z Physics at LEP 1, Geneva 1989 (CERN, Geneva, 1989), Vol. 1, pp. 373-453 and Zuerich Tech. Hochsch.-ETH-PT-89-39.

[2] A. Signer and L. Dixon, Phys. Rev. Lett. 78, 811 (1997); Z. Nagy and Z. Trócsányi, Phys. Rev. Lett. 79, 3604 (1997); J. M. Campbell, M. A. Cullen, and E. W. N. Glover, Eur. Phys. J. C 9, 245 (1999); S. Weinzierl and D. A. Kosower, Phys. Rev. D 60, 054028 (1999).

[3] Z. Nagy and Z. Trócsányi, Phys. Rev. D 59, 014020 (1999); 62, 099902(E) (2000).

[4] R. Frederix, S. Frixione, K. Melnikov, and G. Zanderighi, J. High Energy Phys. 11 (2010) 050.

[5] S. Becker, D. Gotz, C. Reuschle, C. Schwan, and S. Weinzierl, Phys. Rev. Lett. 108, 032005 (2012).

[6] A. Gehrmann-De Ridder, T. Gehrmann, E. W. N. Glover, and G. Heinrich, J. High Energy Phys. 11 (2007) 058; 12 (2007) 094.

[7] S. Weinzierl, J. High Energy Phys. 06 (2009) 041; 07 (2009) 009.

[8] S. Catani, L. Trentadue, G. Turnock, and B. R. Webber, Nucl. Phys. B407 (1993) 3; A. Banfi, H. McAslan,
P. F. Monni, and G. Zanderighi, J. High Energy Phys. 05 (2015) 102; T. Becher and M. D. Schwartz, J. High Energy Phys. 07 (2008) 034; A. H. Hoang, D. W. Kolodrubetz, V. Mateu, and I. W. Stewart, Phys. Rev. D 91, 094017 (2015); T. Gehrmann, G. Luisoni, and H. Stenzel, Phys. Lett. B 664, 265 (2008).

[9] G. Somogyi, Z. Trócsányi, and V. Del Duca, J. High Energy Phys. 06 (2005) 024; 01 (2007) 070; G. Somogyi and Z. Trócsányi, J. High Energy Phys. 01 (2007) 052; 08 (2008) 042; U. Aglietti, V. Del Duca, C. Duhr, G. Somogyi, and Z. Trócsányi, J. High Energy Phys. 09 (2008) 107; P. Bolzoni, S. O. Moch, G. Somogyi, and Z. Trócsányi, J. High Energy Phys. 08 (2009) 079; P. Bolzoni, G. Somogyi, and Z. Trócsányi, J. High Energy Phys. 01 (2011) 059; V. Del Duca, G. Somogyi, and Z. Trócsányi, J. High Energy Phys. 06 (2013) 079; G. Somogyi, J. High Energy Phys. 04 (2013) 010.

[10] L. W. Garland, T. Gehrmann, E. W. N. Glover, A. Koukoutsakis, and E. Remiddi, Nucl. Phys. B627, 107 (2002); B642, 227 (2002).

[11] E. W. N. Glover and D. J. Miller, Phys. Lett. B 396, 257 (1997); Z. Bern, L. J. Dixon, D. A. Kosower, and S. Weinzierl, Nucl. Phys. B489, 3 (1997); J. M. Campbell, E. W. N. Glover, and D. J. Miller, Phys. Lett. B 409, 503 (1997); Z. Bern, L. J. Dixon, and D. A. Kosower, Nucl. Phys. B513, 3 (1998).

[12] K. Hagiwara and D. Zeppenfeld, Nucl. Phys. B313, 560 (1989); F. A. Berends, W. T. Giele, and H. Kuijf, Nucl. Phys. B321, 39 (1989); N. K. Falck, D. Graudenz, and G. Kramer, Nucl. Phys. B328, 317 (1989).

[13] V. Del Duca, C. Duhr, G. Somogyi, F. Tramontano, and Z. Trócsányi, J. High Energy Phys. 04 (2015) 036.

[14] S. Brandt, C. Peyrou, R. Sosnowski, and A. Wroblewski, Phys. Lett. 12, 57 (1964).

[15] E. Farhi, Phys. Rev. Lett. 39, 1587 (1977).

[16] G. Parisi, Phys. Lett. B 74, 65 (1978).

[17] J. F. Donoghue, F. E. Low, and S. Y. Pi, Phys. Rev. D 20, 2759 (1979).

[18] D. P. Barber et al., Phys. Rev. Lett. 43, 830 (1979).

[19] C. L. Basham, L. S. Brown, S. D. Ellis, and S. T. Love, Phys. Rev. Lett. 41, 1585 (1978).

[20] S. Weinzierl, Eur. Phys. J. C 71, 1565 (2011); Eur. Phys. J. C 71, 1717(E) (2011).

[21] J. Alwall, R. Frederix, S. Frixione, V. Hirschi, F. Maltoni, O. Mattelaer, H.-S. Shao, T. Stelzer, P. Torrielli, and M. Zaro, J. High Energy Phys. 07 (2014) 079. 